Academic Platform Journal of Engineering and Science

\title{
Döner (Spin) Kaplama ile İki Boyutlu Polimerik Nanokompozitlerin Geniş Alanlı Üretimleri
}

\author{
*Numan Gözübenli \\ Moleküler Biyoloji ve Genetik Bölümü, Harran Üniversitesi, Şanlıurfa, Türkiye, gnuman @ harran.edu.tr, \\ Araştırma Makalesi \\ Geliş Tarihi: 14.06.2019 \\ Kabul Tarihi: 14.12.2019
}

$\ddot{O} z$

Bu çalışmanın amacı, döner-kaplama tekniği kullanılarak yüksek kristal kalite kolloidal şablonların eldesi ve bu şablonlardan üretilen nanokompozitlerin üretim koşullarının belirlenmesidir. $\mathrm{Bu}$ amaçla, hazırlanan akrilat monomeri içerisine homojen dağılmış silika kolloidleri kullanarak çeşitli yüzeyler üzerine döner kaplama tekniği ile cam yüzeyler kaplandı. Merkez kaç kuvvetlerine dayalı bu kaplama tekniği ile yüksek kaliteli nanodizilimler ve polimerizasyon aşamasıyla, iki boyutda kolloidal kristallerin polimer yapıları başarılı bir şekilde hazırlandı. Etanolde, seyreltik silika nanoküreler temizlendikten sonra viskozitesi 60 cps olan etoksile trimetilolpropan triakrilat monomer ile hacimsel fraksiyonları $<\% 20$ olacak şekilde çözüldü. Hazırlanan bu kolloidal süspansiyon-monomer karışımı cam yüzeyler üzerine oldukça tek dizilimli olarak kaplandı. Filmin kalınlığı sadece dönüş hızı ve dönüş zamanı değiştirilerek kontrol edilebilmektedir. Polimer matrisinin ve silis kürelerinin seçici olarak uzaklaştırılması, reaktif iyon aşındırıcı ve hidroflorik asit uygulamalarıyla gerçekleştirilmiş olup, sırasıyla geniş alanlı kolloidal kristallerin makro gözenekli polimer şablonları elde edildi. Görünür ve yakın kızılötesi bölgelerdeki normal iletim spektrumları, iki boyutlu bu nanoyapılardan, Bragg kırınımın belirgin tepe noktaları grafiklerle belirlendi. Optik disk ölçekli kaplama işlemlerine dayalı bu teknik, standart yarı iletken mikrofabrikasyonlara ve optik biyosensör üretimine uyumludur. Döner kaplama işleminin, merkez kaç kuvvetine dayalı kristalleşme uygulaması, kaplama tekniklerine dayalı, teknolojik uygulamalarda rahatlikla kullanılabilmektedir.

Anahtar Kelimeler: Döner (spin) kaplama, kolloidal litografi, kolloidal silika kristali, fotonik kristal, nanodizilimler

\section{Mass Fabrication of Two Dimensional Polymeric Nanocomposites with Spin Coating}

\author{
*Numan Gözübenli \\ Molecular Biology and Genetic Department, Harran University, Şanlıurfa, Turkey, gnuman@ harran.edu.tr
}

\begin{abstract}
The aim of this study is to obtain high crystal quality colloidal templates using spin-coating technique and to determine the production conditions of nanocomposites produced from these templates. For this purpose, the glass surfaces were coated by spin- coating technique on various surfaces using homogeneously dispersed silica colloids in the prepared acrylate monomer. With this coating technique based on centrifugal forces, and following polymerization step, high quality nanoarrays of colloidal crystals in two dimensions were successfully prepared. In ethanol, the dilute silica nanospheres were removed and then dissolved with ethoxylated trimethylolpropane triacrylate monomer having a viscosity of $60 \mathrm{cps}$ to $<20 \%$ volumetric fractions. This prepared colloidal suspension-monomer mixture was coated on glass surfaces in a very uniform manner. The thickness of the film can only be controlled by changing the rotation speed and time of spin coating. Selective removal of the polymer matrix and silica spheres was achieved by reactive ion abrasive and hydrofluoric acid treatments, yielding large area colloidal crystals and macroporous polymers, respectively. The normal transmission spectra in the visible and near infrared regions and the apparent peaks of Bragg diffraction from these two-dimensional nanostructures were determined by graphs. Based on optical disc-scale coating, this technique is compatible with standard semiconductor microfabrications and optical biosensor production. Crystallization application based on centrifugal force of spin coating process can be easily used in technological applications based on coating techniques.
\end{abstract}

Keywords: Spin coating, colloidal lithography, colloidal silica crystal, photonic crystal, nanoarrays 


\section{GíRiş}

Kolloidal litografi (Nanolitografi) teknikleri kullanılarak optik dalga boyu aralığında üç boyutlu (3B) kolloidal fotonik kristaller üretilmektedir[1]. Kendinden organize olabilen "ters opaller" olarak adlandırılan bu yapay kolloidal kristaller, 3B şablonların makro gözenekli malzemelerinde yaygın olarak kullanılmaktadır[2-4]. Karmaşık fotolitografi tekniklerine kıyasla, temelde kolloidal litografi; çok daha basit, hızlı ve ucuz bir yaklaşım sunduğu için, son zamanlarda bilim insanlarının dikkatini üzerine çekmiş̧ir. Milimetrik boyutlarda tekli veya çoklu kristal tabakalar; sedimantasyon[5], elektrostatik itme[6, 7], şablon destekli montaj[8,9], fiziksel sinırlandırma[10], kilcal kuvvetlere dayalı kendinden montaj[11, 12], ve elektrik alan montajı[13] gibi çeşitli yöntemler kullanılarak, günler hatta haftalarla ifade edilen süreçlerde[14] hazırlanabilmektedir. Nanolitografide kullanılabilen bu teknikler; düşük verimli olduklarından kitlesel üretimler için elverişsizdir. Ancak, endüstriyel ölçekli bir kitlesel üretime dönüştürülebilmeleri, yarı iletken endüstrinin seri mikro üretimi için, can sıkıcı ve olanaksız görülmektedir[15-17]. Bu yöntemler; geniş alan üzerinde, homojen olmayan ve kontrol edilemeyen farklı kristal kalınlıkları oluşturmaktadır. Aynı zamanda, buna benzer engeller, teknolojik açıdan önem arz eden iki farklı malzemenin, yani makro gözenekli polimer ve polimerik nanokompozitlerin üretim yöntemlerinide zorlaştırmıştır $[1$, 18].

Mükemmel periyodik sıralamaya sahip nanokompozitlerin kolloidal kristal tabanlı santimetre boyutunda üretimleri ile karşılaştırıldığında bu diziler; yukarıdaki klasik teknikler kullanılarak sadece mikron boyutlarında elde edilebilmektedir[19, 20]. Temas boşlukları olan bu yüksek dereceli polimerik dizilerin; makromoleküller ve DNA ayrımı için ayırma ortamı[21, 22], biyosensör[23], ve "kolloid kristalleri" oluşturmak için "lostwax" iskeleleri olarak başarılı bir şekilde uygulanabildiği kanıtlanmıştır[24, 25]. Ayrica, bu nanokompozit sistemler dielektrik malzemelerde sinyal geciktirici ve entegre devrelerdeki ara bağlantılarda gürültü oranlarını azaltıcı yenilikler sunmaktadır[26]. Polimere gömülü kolloidal kristallerle (veya 3B dizilimli polimerik nanokompozitler), fotonik kağıtlardan ultra yüksek yoğunluklu optik kayıt malzemelerine kadar önemli teknolojik uygulamalarda, başarı sağlanmıştır[16, 27]. Fakat, bu nanokompozit yapıların üretiminde kullanılan elektrostatik itme[28, 29] hibrit çekirdek yaklaşımları, kristal kalınlığının kontrolünde, hem zorluklar barındırır, hem de çok zaman kaybettirir[3032].

Pratikte, geniş alanlı, düzlemsel numunelerin kontrol edilebilir kalınlıkta kitlesel üretimine izin veren bir teknikle geliştirilmesi, oldukça arzu edilen bir araştırma konusudur. $\mathrm{Bu}$ amaçla; döner (spin) kaplama tekniği, kolloidal litografide 3B kolloidal kristal şablonlarının geliştirilmesinde yaygın olarak tercih edilen ilk teknik olmuştur[6, 33]. Bu teknik, 3B kristallerin geniş alanlarda kolay ve hızlı bir şekilde üretimlerini gerçekleştirmiştir[12]. Destekleyici yüzeyler üzerindeki kolloidal süspansiyon viskozitesinin arttırılması gibi yöntemlerle, 3B kolloidal kristallerin sıralı dizilimleri ve hassasiyet derecesi oldukça arttırılmıştır[34]. Bununla birlikte, döner kaplama göründüğünden çok daha karmaşık bir işlem olup, son zamanlarda homojen dağılımlı kolloidlerden oluşan 1B [34, 35], 2B [36, 37], ve 3B [14, 24] periyodik yapılar elde edilmiştir $[38,39]$. Ancak yukarda belirtilen çalışmalarda, kolloidal tabakaların transfer yöntemi kullanmadan, direk olarak döner kaplamayla, (tek tabakal1) 1B veya 2B kristal tabaka üretimleri henüz gerçekleştirilememiştir[ $[8,23,40]$. $\mathrm{Bu}$ çalışmanın amacı; döner kaplamayla cam yüzey üzerinde kolloidal silika tabakalarının, direk olarak 2B nanoyapıların üretimlerini geniş alanda gerçekleştirmek ve 2B nanokompozitlerin pratik üretimini sağlayan koşulları belirlemektir.

\section{MALZEME VE YÖNTEM}

\subsection{Malzemeler ve Yüzeyler}

Tüm solventler ve kimyasallar, reaktif kalitede olup, daha fazla saflaştırma gerektirmeden kullanıldı. Etanol (200), pharmaco adlı firmadan satın alındı. Moleküler ağırlığı 428 $\mathrm{g} / \mathrm{mol}$, viskozite $60 \mathrm{cps}$ olan etoksile trimetilolpropan triakrilat monomer (ETPTA, SR 454) Sartomer'den sipariş edildi. Foto başlatıcı olarak, Darocur 1173 (2-hidroksi-2metil-1-fenil-1-propanon), Ciba-Geigy marka kullanıldı. Cam diskler, 3-akriloksipropil triklorosilan (APTCS), United Chemical Technologies'den tedarik edildi. Tetrabutilamonyum klorür, Aldrich'ten satın alındı. 6 adet cam disk (600 nm termal oksit tabakalı, Wafernet, San Jose, CA) "Piranha" çözeltisinde (3: 1 konsantre sülfürik asit karışımı) $20 \mathrm{dk}$ bekletildikten sonra yarım saat boyunca $\% 30$ hidrojen peroksit ile oksitlenerek temizlendi. Daha sonra Milli-Q su ile durulanarak $\left(0.05 \mu \mathrm{S} \mathrm{cm}^{-1}\right)$ azot gazı altında kurutuldu. Temizlenmiş cam yüzeyler, daha sonra yüzeyler üzerine APTCS sürülerek astarlandı. Q-uçlu pamuk çubuk ile astarlama sonrası, iki kez 200'lük etanol ile durulanıp, sıcak bir plaka üzerinde $110^{\circ} \mathrm{C}$ de iki dakika boyunca isıl işleme maruz birakıldı.

\subsection{Kolloidal Dispersiyonların Hazırlanması}

Sentez, saflaştırma işlemleri, 200' lük etanolde ağırlıkça \% 5 'den küçük homojen silika nanokürelerin hacimsel fraksiyonları; Dr. Jiang ve meslektaşlarının son yıllardaki çalışmaları referans alınarak hazırlandı [22, 33]. Hesaplanan miktarda saflaştırılmış silika çözeltisinin santrifüj edilmesinin ve süpernatan çözücünün atılmasının tamamlanmasindan sonra silis kolloidleri; Thermdyne marka (3200) karıştırıcı kullanılarak ETPTA monomerleri homojen hale getirildi. \% 1 (ağırlık) Darocur 1173, bu karışıma, foto başlatıcı olarak eklendi. Nihai parçacık hacminin oranı, yaklaşı \% 20 olarak belirlendi. Şeffaf ve yapışkan solüsyon, kalan etanolün buharlaşması için gece boyunca açık bir şişe içinde beklemeye bırakıldı. 


\subsection{Deneysel Araçlar}

Taramalı elektron mikroskobu ve EDAX görüntüleri için Zeiss-SEM Evo50 SEM cihazı kullanıldı. Silika kolloidleri, ETPTA monomeri içerisinde homojenize edebilmek için Thermdyne marka (3200) karıştırıcı kullanıldı. SilikaETPTA dispersiyonunu farklı malzemeler üzerine kaplamada standart bir döner kaplayıcı (Laurell H6-23) cihaz kullanıldı. ETPTA monomer kaplı cam yüzeyler, polimerizasyon için darbeli bir UV (UVP Compact ve Manuel UV lamba) kürleme sistemine aktarıldı. ETPTAkolloid dispersiyonu, 4 saniye boyunca UV radyasyonuna maruz bırakılarak hızlı bir şekilde polimerize edildi. Polimerleştirilmiş nanokompozit film kalınlığını ölçmek için girişim bazlı film kalınlığı, ölçüm cihazı (F20, Filmetrics) kullanıldı. Kolloidal kristallerin açığa çıkarılması ve polimer ETPTA 'nın uzaklaştırılması için bir Quartz Serisi, Anatech oksijen plazma aşındırıcı kullanıldı. Absorpsiyon ve iletim (transmission) spektrumları sonuçları, bir Ocean Optics ST2000 fiber optic UV-vis-NIR spektrometresi kullanılarak değerlendirildi.

\subsection{Kolloidal Süspansiyonların Döner Kaplanması ve Fotopolimerizasyon}

Silika-ETPTA çözelti karışımının $500 \mu \mathrm{L}$ lik hacmi, 6 adet cam malzeme üzerine aktarılarak, kaplama işleminden sonra yüzey üzerine yayılımı sağlamak vede çözeltiyi tüm yüzeye yaymak için disk yüzeyler 200 rpm' de döner-kaplayıcı üzerinde bir dakika boyunca döndürülerek kaplandı. Altı kollu kırınım yıldızı yaklaşık 30 saniyede oluştu. Kaplanacak yüzey daha sonra istenen hizda (2000 rpm), hedef kalınlığa ulaşmak için belirli bir süre boyunca döndürüldü. Etanol, kaplama fazlası polimer matriks sökme işleminde kullanıldı. Döner kaplamadan sonra, kaplanan yüzey bir vakum odasına aktarılarak $30 \mathrm{~s}$ içinde 0.1 Torr bir vakumda bekletildi. Ardından UV lambasında 2 dakika UV ışı̆̆ına maruz bırakılarak polimerize edildi.

\subsection{Reaktif İyon Aşındırma (RİA)}

Dört saniye boyunca darbeli bir UV compact sistemi içerisinde UV radyasyonuna maruz kaldıktan sonra, açığa çıkmamış, atık monomer ve silika kolloidler, alkolde durulama yoluyla uzaklaştırıldı. Cam yüzeyler üzerine kaplanan polimer matriks numuneler daha sonra tam polimerizasyon için UV lambasında 1-2 dk bekletildi. 30 mTorr oksijen basıncında, $50 \mathrm{sccm}$ akış hızında ve $500 \mathrm{~W}$ gücünde çalıştırılan oksijen reaktif iyon aşındırıcı (Unaxis) ETPTA'yı aşındırmada kullanıldı. 30 mikron kalınlığındaki bir numunenin kaplanmamış ETPTA'sının tamamen uzaklaştırılması yaklaşı 4 dakika sürdü. Açıkta kalan silika küreler daha sonra 40 saniye süreyle $\% 1$ hidroflorik asit çözeltisinde bekletildi.

\subsection{Numune karakterizasyonu}

Taramalı elektron mikroskobu ve EDAX sonuçları ZeissSEM Evo50 SEM 'de alındı. Görüntüleme öncesi, ince bir altın/krom tabakası (yaklaşık 5-8 nm) örnekler üzerine kaplandı. Elde edilen numunelerin optik özellikleri, normal oranlarda iletim (transmission) spektrumları bir Ocean Optics UV-vis-NIR spektrometresi kullanılarak değerlendirildi.

\section{BULGULAR}

Süspansiyonların döner kaplama cihazı üzerindeki işlemi daha önceki 2.3. ve 2.4. kısımda bahsedilen metotlar izlenerek gerçekleştirildi. Düzgün silika kolloidleri viskoz ve uçucu olmayan monomer etoksile trimetilolpropan triakrilatta (ETPTA), \% 19.8'lik parçacık ağırlığının fraksiyonu ile \% 1 Darocur 1173 foto başlatıcı karıştırılarak yüzeye kaplandı. Silis kürelerinin ekstra çözücüye ve cam yüzeylerin modifikasyonuna ihtiyaç duyulmadı.

Elde edilen saydam kararlı küçük küre süspansiyonları en az altı ay $(<400 \mathrm{~nm})$; buna karşılık daha büyük kürelerden oluşan süspansiyonların 1 ila 3 aya kadar çözelti stabilitesini koruduğu gözlemlendi. Ancak süspansiyonların sonikasyonla kolayca dağılabildiği ve kristallerin 1-2 saat içerisinde çökeldiği gözlemlendi. Beyaz ışık altında, güçlü bir yansıma sağlayan ve kaplama süreci öncesi oluşan yanardöner polikristal görünüm, altı kollu kırınım yıldızı yaklaşık 30 sn içerisinde (Şekil 1-(solda) ve Şekil 2) oluştu.
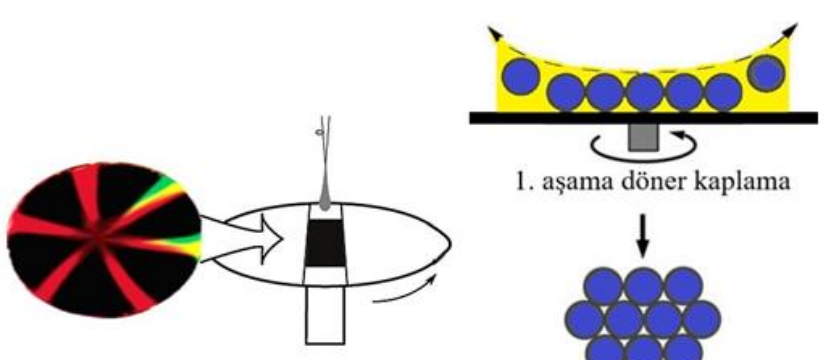

1. aşama döner kaplama

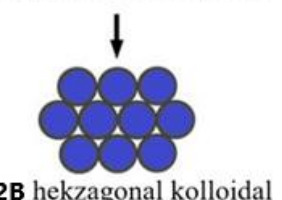

2B hekzagonal kolloid kristal dizilim

Şekil 1. Solda, 2B ve 3B yapılı nanokompozit dizilimde kullanılan döner kaplama sürecinin şematik gösterimi ve hazırlanan 6 kollu kristal yapının resmi. Sağda ise 1. aşama kaplamada elde edilen 2B hekzagonal dizilim süreci şematize edildi.

Kırınım yıldız formunun bitişik kolları tam olarak $60^{\circ}$ lik açıya sahip olup, malzeme yüzeyine paralel kürecikler, altıgen olarak (hekzagonal) paketlendiği gözlemlendi. Küreler tarafindan oluşturulan kristal iskelet, döner kaplama sırasında kararlıdır. Küreleri çevreleyen akışkan, viskoz olup, hizalanan kristaller, kaplama durduktan sonra da, kararlılığını sürdürdü. Monomerler daha sonra UV radyasyona maruz kalarak polimerize edildi. 


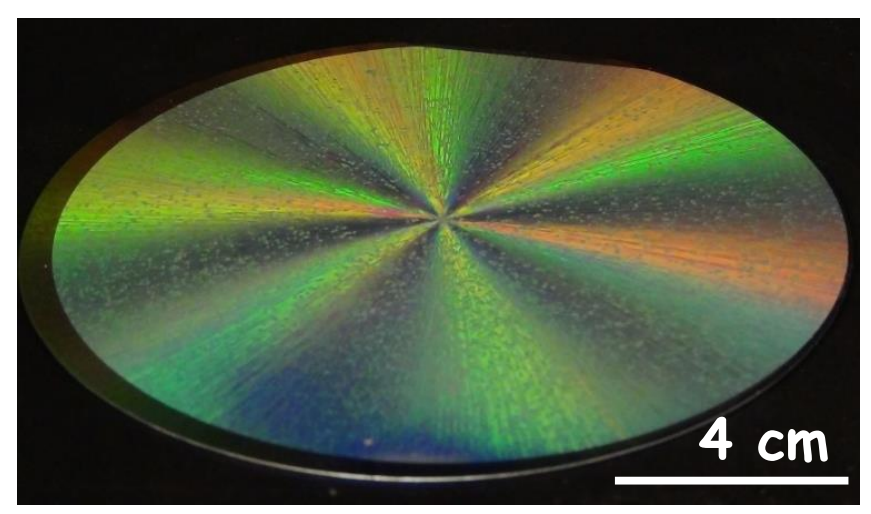

Şekil 2. $15 \mathrm{~cm}$ lik bir silikon wafer üzerine kaplanmş nanokompozit filmin beyaz ışıkla aydınlatılmış fotoğrafi. $325 \mathrm{~nm}$ çapında kolloidal kürelerle kaplanan yüzey, 20 saniye süreyle 6000 rpm'de döndürülerek elde edildi. Numune, kaplama sonrası polimerizasyon aşaması ve onu takiben 60 saniyelik oksijen iyon aşındırmaya maruz kaldı. Kendiliğinden parıldayan 6 kollu gökkuşağı lüminesans özelliği kazandı.

\subsection{ETPTA Polimer Matrisinin ve Silika Kürelerinin Seçici Uzaklaştırılması}

Gömülü kolloidal kristalleri açığa çıkarmak ve ETPTA polimer matrisi uzaklaştırmak için (Şekil 3A ve 3B), 1 Torr oksijen basıncında, $450 \mathrm{sccm}$ oksijen akış hızı ve $500 \mathrm{~W}$ da çalışan bir oksijen plazma aşındırıcı (Quartz Serisi, Anatech) uygulandı. 30 mikron kalınlığındaki bu polimer matrislerin tamamen uzaklaştırılması yaklaşık 4 dakika sürdü. Makro gözenekli polimerleri ve kolloidal parçacıkların şablonlarını açığa çıkarabilmek için, yukarda bahsedilen oksijen plazma aşındırıcının aynı koşulları 10 saniye için uygulandı. Silika küreciklerini görünür kılmak ve üst tabakaları ortaya çıkarmak için yüzey ETPTA polimer tabakası kısmen uzaklaştırıldı (Şekil 4A ve 4B). Bu süreçde; makro gözenekli polimerlerin (şablonların) hekzagonal dizilimleri ve gözenekliliği etkilenmedi. Ayrıca, silika şablonlarını elde etmek için $\% 2$ hidroflorik asit sulu çözeltisi kullanıldı.

Sentezlenmiş kolloidal kristal polimer nanokompozit film, parlak monokromatik kırınım sergiledi (bakınız Şekil 1 ve 2). Bir taramalı elektron mikroskobu (SEM) ile üstten görüntüsü (Şekil $3 \mathrm{~A}$ ve $3 \mathrm{~B}$ ) ve Fourier dönüşümleri (FFT, Şekil 3A üzerinde), film üzerinde altıgen dizilimi içeren sıralı yapıları gösterildi. Daha yüksek büyütme yapıldığında (Şekil 3A), üst polimer tabaka iyon aşındırıcı ile uzaklaştırıldığındaki SEM görüntüsü (Şekil 4A ve 4B) başka ilginç bir özellik olan üst katman kürelerinin birbirine dokunmadığı ancak mesafenin 1.41D olarak partiküller arası sabit bir mesafenin olduğu gösterildi. Partiküller arasındaki mesafede kullanılan D, kolloidal partiküllerin çapıdır. Yakın dizilimli kolloidlerin, polimer matrisinin oksijen plazma aşındırmayla seçici olarak uzaklaştırılmasından sonra hem SEM görüntüsünde, hemde fotoğraflarda daha belirgin hale getirildi. 60 sn lik plazma aşındırma işlemi sonrası Şekil 2, 4A, 4B, 5B ve 5C deki SEM görüntüleri elde edildi. 15 cm'lik bir örnek üzerinde yapılan kapsamlı SEM ve Fourier dönüşümü (FFT) sonrası, kristalografik yönelimdeki sapmaların $10^{\circ}$ 'den daha az olduğu ortaya kondu (Şekil 3A). Tabakaların düzlemsel ve dikey sıralamalı dizilimleri, üstten ve yan kesit görüntülerle ortaya çıkarıldı (bkz. tek tabaka için Şekil 5A, 5B, çift tabakalar için Şekil 5C ve çok tabakalar için Şekil 5D).

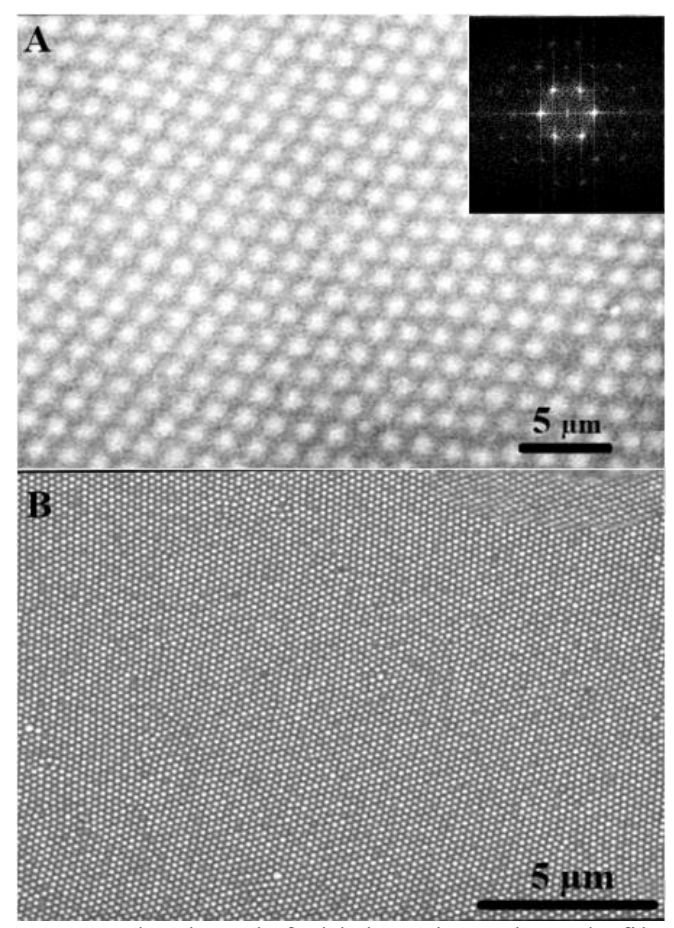

Şekil 3. Döner kaplamalı farklı küre boyutlarında filmlerden elde edilen gömülü kolloid nanokompozitlerin tipik üstten SEM görüntüleri. (A) $1530 \mathrm{~nm}$ çapındaki küreler ve 600 rpm'de 120 saniye boyunca kaplanmış bir numune. (B) 130 $\mathrm{nm}$ çapındaki kürelerden hazırlanan ve $600 \mathrm{rpm}$ 'de $120 \mathrm{~s}$ boyunca kaplanan bir numune.

Uygulanan spin kaplama ve fotopolimerizasyon süresi (dakika olarak) göz önüne alındığında, zorlu sedimantasyon süreci tamamen engellendi. Dönüş kaplama tekniğini kullanarak, $100 \mathrm{~nm}$ ila $2 \mu \mathrm{m}$ arasında geniş çaplara sahip silika kolloidleri yardımıla, 2 boyutlu ve 3 boyutlu nanokompozit filmler elde edildi. $130 \mathrm{~nm}$ (bkz. Şekil 3B) ve $1530 \mathrm{~nm}$ (bkz. Şekil 3A) çaplı kolloidal parçacıklardan yapılan iki kristal tabaka örneği, 325 nm'lik küreler (bkz. Şekil 4A) ile yapılan kristal tabakadaki benzer uzun skala dizilimlerdeki gibi (yaklaşık 1.41D), merkezden merkeze olan uzaklığın sabit kaldığı gösterildi. 


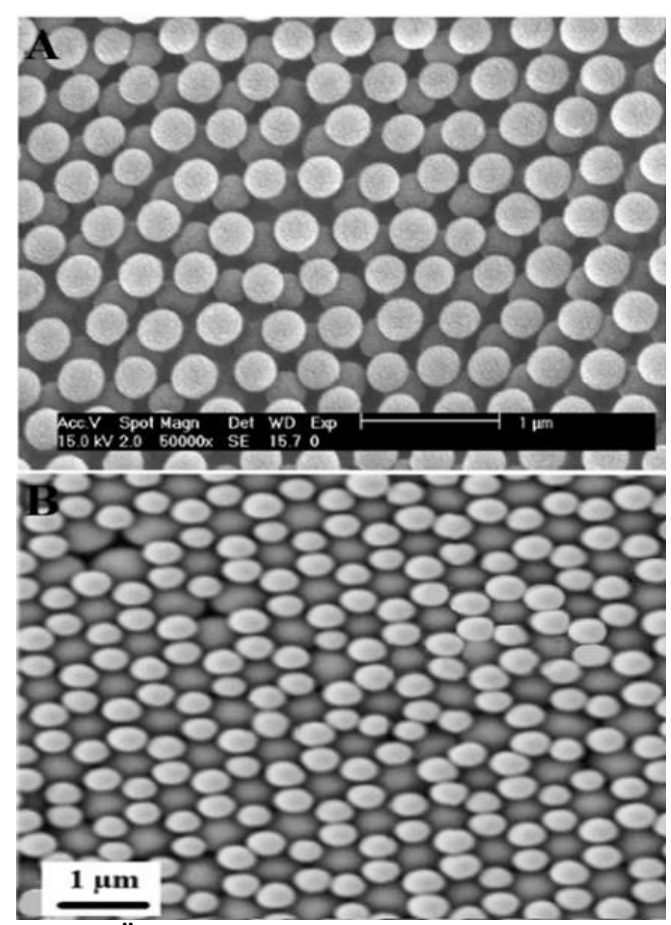

Şekil 4. A) Üst polimer matrisin reaktiv iyon aşındırıcı yardımıyla seçici olarak uzaklaştırılmasıyla, optik disk ölçeğinde (15 cm çaplı) 3B kolloidal kristal (325 nm çaplı) dizilimlerin görüntüsü. B) 1. aşama döner kaplama sonrası, ikinci tabaka kolloidlerin kaplanıp, polimer tabakanın reaktiv oksijen iyonu ile uzaklaştırılması sonucunda (3B kristal dizilimi) elde edildi.

Döner kaplı nanokompozit filmler, film kalınlığı ölçüm sistemi (F20, Filmetrics) tarafindan belirlenen $15 \mathrm{~cm}$ çapındaki bir yüzey üzerinde \% 4'ten daha düşük bir sapmayla kaplandığı belirlendi. Formüle edilen film kalınlığı, (tek katmandan yüzlerce kolloidal katmana kadar) kaplama hızını ve süresini değiştirerek kolayca kontrol edilebildi (Şekil 5). Kaplama esnasında kaplama cihazının programına (girilen) uygulanan hız, dönüş zamanının karekökü ile ters orantılı olduğu görüldü (Şekil 7A). Bu kaplama tekniğinde çözücüsüz sıvıların spin kaplama modeli ile uyum içinde olup; $\mathrm{H} \approx(\mathrm{A} / \omega \mathrm{xt})$ tahmini yapılarak, Şekil 7 de elde dilen, ters orantı ilişkileri açıklandı. Burada $\mathrm{H}$; film kalınlığı, A; çözeltinin viskozitesi ve yoğunluğu ile belirlenen bir sabittir. $\omega$ ve t; son dönüş hızı ve zamanıdır. Şekil 7 ye göre; $\mathrm{H}$ mikron cinsinden, $\omega$, rpm ve t'nin saniye olarak kabul edildiğinde, yukarıdaki denklemde $\mathrm{A} \approx 97800$ olarak bulundu. Farklı kaplama koşulları hız ve dönüş kaplama hızının; hazırlanmış üç farklı kolloidal katman içeren (Şekil 5A ve 5B) tek katman, (Şekil 5C) iki katman, ve (Şekil 5D) 7 katmanlı elde edilen kolloidal kristallerin tabaka sayısı ile doğrudan ilişkili olduğu görüldü.

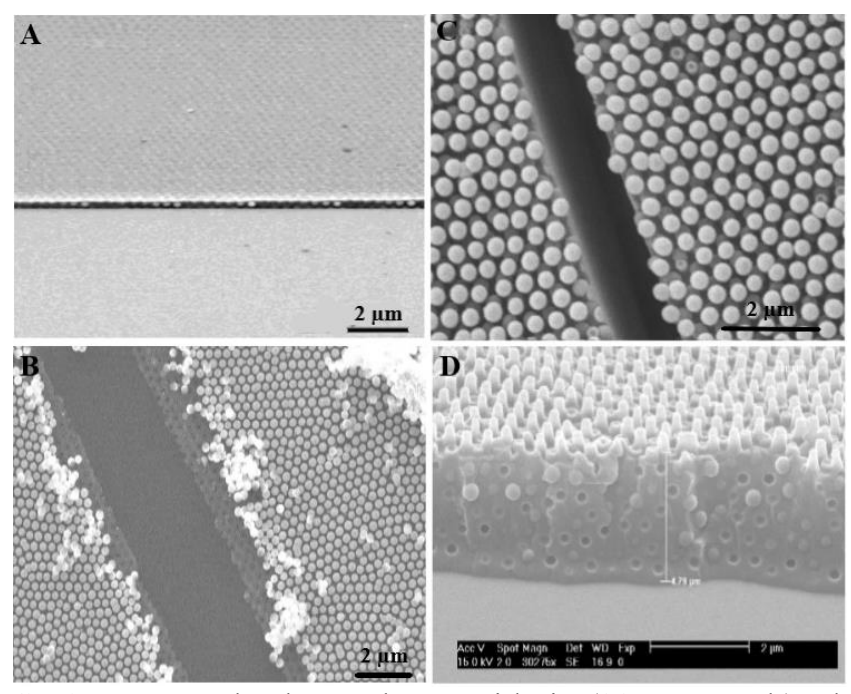

Şekil 5. Döner kaplı nanokompozitlerin (325 nm çaplı) tek tabakalı 2B (Şekil 5A, 5B) ve çok tabakalı 3B (sağda Şekil $5 \mathrm{C}$, 5D) filmlerin yapıları, tipik üstten ve yandan SEM görüntüleri ile ortaya kondu. (A) 6000 rpm'de 120 sn. sürede kaplanarak elde edilen filmin yandan SEM görüntüsü (B) Oksijen iyon aşındırıcı ile polimer tabakanın uzaklaştırılması sonrası tek katmanlı kolloidal kristal dizilimin üstten bir SEM görüntüsü. Tek tabaka olduğunu kanıtlamak için, numune yüzeyine jilet çiziğini gösteren üstden SEM görüntüsü. (C) $6000 \mathrm{rpm}$ 'de $1 \mathrm{dk}$. sürede kaplanmış 2 katmanlı, oksijen iyonu ile aşındırılarak üst polimer tabakanın uzaklaştırılmış, üstten SEM görüntüsü. (D) 325 nm çaplı kolloidlerin polimer matrisin $30 \mathrm{~s}$ sürede 4000 rpm'de kaplanarak, 7 katmanlı (üst polimer tabaka uzaklaştırılmış) kristalin yandan görüntüsü.

\subsection{Film Kalınlığının Döner Kaplama Hızı ve Kaplama Süresiyle Kontrolü}

Kaplama sürecinden sonra, polimerleştirilmiş nanokompozit film kalınlıkları; girişim tabanlı bir (F20, Filmetrics) cihazı ile 6 örnek üzerinden, her birinden 6 farklı noktadan ölçüm alındı. Nanokompozit filmin ortalama kırılma indisleri (n: 1.433) farklı kalınlıktaki 6 adet örnekten; Metricon 2010 kırılma indisi ölçüm sistemi kullanılarak elde edildi. Film kalınlığının nihai kaplama hızı ile ilişkisini belirlemek için (Şekil 7A), sabit kaplama süresi, $120 \mathrm{~s}$ de, farklı kaplama hizlar1 300,600, 1200, 2000, 4000 ve 6000 rpm'de $15 \mathrm{~cm}$ boyutlarında, 6 adet cam yüzey bu kaplama programı uygulanarak kaplandı. Numunelerdeki kalınlık 6 rasgele nokta seçilerek ölçüldü ve ortalama film kalınlığı belirlendi. 6 ayrı noktada homojen kalınlığa sahip olmayan (kalınlık sapma > \% 4) numuneler belirlenerek bunlar yerine sapma değeri \% 4 ün altında olanlar, kolloidal film şablonları için seçildi. Film kalınlığının son kaplama süresi ile ilişkisinin belirlenmesinde, sabit kaplama hızı için, farklı kaplama zamanlarında, 30, 90, 120, 240, 480, ve 960 s'de 6 adet numune 600 rpm sabit hızda kaplandı (Şekil 7B). 


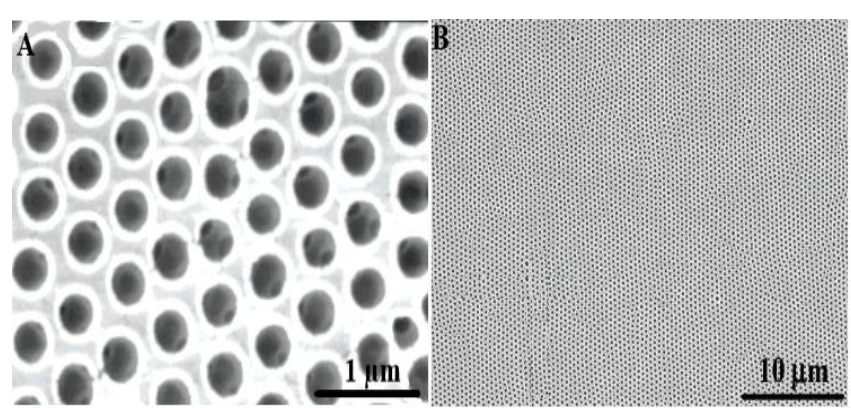

Şekil 6. HF asit uygulaması sonrası silika kolloidal parçacıklarının uzaklaştırılması sonrası çok tabakalı kristal şablonlarının (ayrıntılı görüntü 6A) membran gözenekli SEM görüntüleri (geniş açıdan 6B).

Kaplama sonrası elde edilen filmler RİA ile yüzey aşındırma uygulanarak (120 sn.) yüzeydeki ilk tabaka kolloidlere ulaşana kadar ETPTA katman uzaklaştırıldı. Daha sonrada kolloidal şablonları açığa çıkarmak ve kolloidal parçacıkları uzaklaştırmak için \%1 lik HF çözeltisine yaklaşık $30 \mathrm{dk}$. bekletilerek kolloidal şablonlar elde edildi (bkz. Şekil 6A ve 6B).
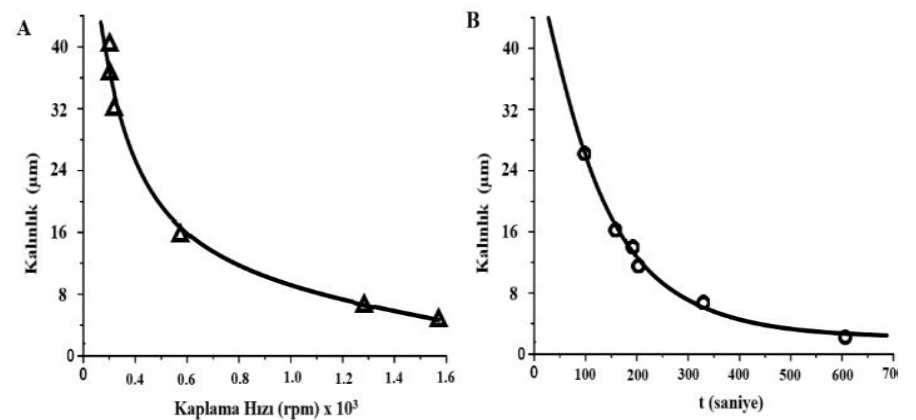

Şekil 7. Kristal kalınlığının hassas kontrolü. Bu durumlar için $325 \mathrm{~nm}$ çapında kolloidler kullanıldı. (A) Sabit kaplama süresi, $120 \mathrm{~s}$ de, doğrusal kaplama hızına karşı kristal kalınlığının ters orantı ilişkisi. (B) Sabit dönüş hızı 600 rpm de, film kalınlığı dönüş süresinin karekökünün tersi ile ilişkisi.

Basit geometrik hesaplama ile, tabakalar boşluğunun $\sqrt{2} / 2 \mathrm{D}$ ye eşdeğer olduğu ve orijinal nanokompozit filmdeki silika parçacıkların hacimsel fraksiyonunun sadece \% 53 lük bir paya sahip olduğu bulundu. Bu olağan dışı açık kristal yapı, normal 1şık altında, görünür yakın IR absorpsiyon spektrumu kullanılarak optik karakterizasyon ile doğruland (Şekil 8).

Şekil 8'de $400 \mathrm{~nm}$ den $560 \mathrm{~nm}$ arasında değişen çaplara ait 4 farklı cam yüzey üzerine kaplanmış silika kürelerinden elde edilen dört makro gözenekli ETPTA film numunesi için optik iletim spektrumları gösterildi. Bragg yasası ile iyi bir uyum içinde olan Şekil 8“de gösterilen liner kırmızı çizgi; boşluk çaplarına karşı $\lambda$ tepe noktası pozisyonlarına denk geldiği görüldü.

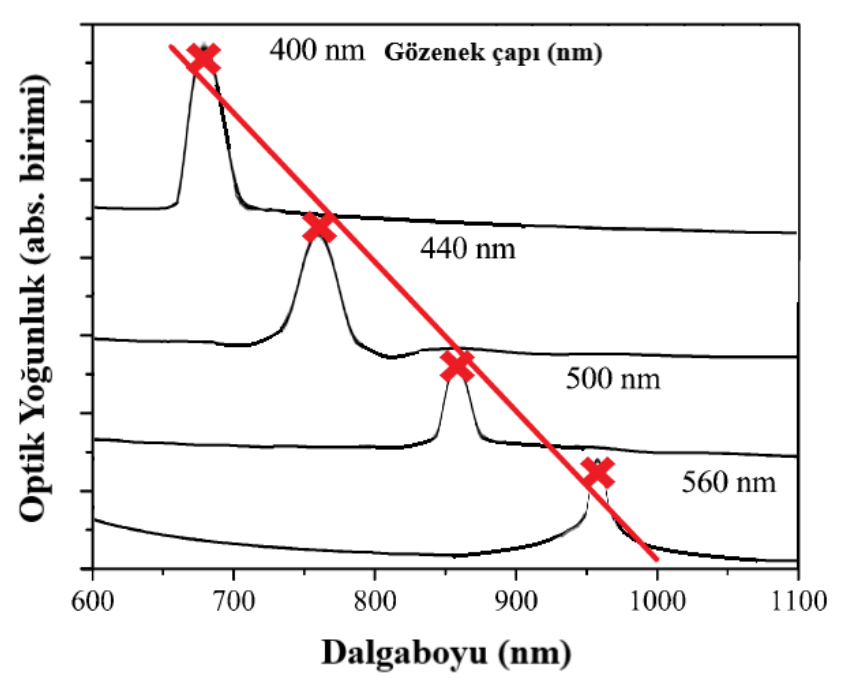

Şekil 8. SEM analizi kullanılarak belirlenen, farklı boşluk çaplarına sahip makrogözenek ETPTA yüzey filmlerinin spektrumu. Kırmızı X noktaları, optik iletim spektrumunun boşluk çapının bir fonksiyonu olarak zirve yaptığı dalga boyunu göstermektedir.

Bu çalışmalar, yüksek merkez kaç kuvvetlerinde kaymakta olan bir katman yapısını ve küçük kuvvetlerde ikiz bir fcc yapısı ortaya koydu.

Silika ETPTA dispersiyonuna $10^{-3} \mathrm{M}$ tetrabutilamonyum klorür ilave edilerek döner kaplama sırasında kolloidal kristalizasyon işlemi üzerindeki tuz etkisi de incelendi. $\mathrm{Bu}$ süreçte, parçacıklar arası elektrostatik itmeyi görebilmek, silika kolloidleride daha "sert küre" haline getirebilmek amaciyla \% $10 \mathrm{v} / \mathrm{v}$ luk silika süspansiyonuna, \% 200 etanol çözücü olarak eklendi. Elde edilen döner kaplı nanokompozit filmler, elektrostatik kuvvetin, kolloidal kristalizasyona, çok küçük katkısı olduğu görüldü. Tuz ilave olmayan örneklerle kıyaslandığında aynı düzlemde parçacıklar arası mesafe $1.41 \mathrm{D}$ olarak bulunmuş ve aynı optik iletim spektrumları sergilediği görüldü.

\section{TARTIŞMA ve SONUÇ}

$\mathrm{Bu}$ çalışmada; basit, hızlı bir döner-kaplama işlemi ile kontrol edilebilen kalınlığa sahip yüksek kaliteli 2B, 3B dizilimli nanokompozitlerin eldesi için, kolloidal kristal ve makro gözenekli polimer deneyleri gerçekleştirilmiştir. Döner (Spin) kaplama, oldukça düzgün kalınlıkta filmler oluşturmada bilinen basit bir kaplama tekniği sunmaktadır[22, 41]. Süspansiyonların döner kaplama cihazı üzerindeki hazırlık süreçleri 2.3. ve 2.4. kısımlarında belirtilen metotlar izlenerek gerçekleştirildi. $\mathrm{Bu}$ açıdan, döner kaplama tekniği, viskoz solüsyonlardan tekrarlanabilir bir şekilde geniş alanlı hekzagonal dizilimli kristallerin eldesini sağlamıştır[26, 42]. 
Çalışmada, yüzey kalınlığının, kaplama hızı ve kaplama süresine olan bağlılığını ortaya koymak için her bir çalışma için, $15 \mathrm{~cm}$ çapında 6 adet cam numune kullanılmıştır. $\mathrm{Bu}$ yüzeyler, dakikalar içinde rutin olarak, kristal kalitesinden, ve hızlı imalat işleminden, ödün vermeden kaplanmıştır. Her bir cam yüzey için en az $3 \mathrm{kez}$ tekrarlama yapılmıştır. $\mathrm{Bu}$ sayade, tekniğin; yüksek oranda ve hızlı bir şekilde tekrarlayarak üretimleri yapabildiği tespit edilmiştir. $15 \mathrm{~cm}$ çaplı düzlemsel kristal kaplamaların yaklaşık $200 \mathrm{~cm}^{2}$ lik alanı kapladığı tespit edilmiştir.

Cam yüzeyler üzerindeki kristal kalınlığı, ortaya çıkan kristalin kalitesini belirlemede önemli bir parametredir. Stöber tekniğine ve McFarland in yapmış oldukları son çalışmalara göz atıldığında bu parametre; hem düzlemsel, hemde kristal kafesin hekzagonal dizilim kalitesini göstermektedir[8, 22,33, 42]. Geniş bir alan üzerinde yüksek oranda homojen ve ayarlanabilir film kalınlığının eldesi, çok tercih edilen bir yöntemdir[1-4]. Silika-ETPTA dispersiyonu çeşitli cam yüzeyler üzerine çeşitli dönüş hıları ve kaplama süreleri, döner kaplama cihazına, sırasıyla programlanarak girildi. Kaplama esnasında oluşan altı kollu yansıma, altıgen paketlenmiş kürelerin Bragg kırınım yansımalarından kaynaklandığı kabul edilmektedir[22, 33-35]. Altı kollu yansıma; kaplama esnasında oluşmakta ve kaplama sonrası polimerizasyon yapılmadığı takdirde, kaplamadan $15 \mathrm{dk}$ sonra merkez kaç kuvveti etkisini yitirmesiyle ortadan kaybolmaktadır. Eğer kaplama tamamlandıktan hemen sonra, $4 \mathrm{sn}$. lik UV radyasyonu altında fotopolimerizasyon sağlanırsa, altı kollu yansıma koruma altına alınmaktadır. Bu koruma, tamamen; dispersiyon haldeki kolloidal kristal yapının polimerizasyonla, katı faz hale getirilmesi ile sağlanmakta[15- 22]. Kaplama yüzeyinde oluşan filmin parlak yanar-döner renkleri, (Şekil 2), üst ve yandan SEM görüntüleri (Şekil 3A ve 3B) ile kolaylıkla seçilebilmektedir. Şekil 3A üzerinde görülen fourier dönüşümü, 3 boyutlu sıralı hava boşluklarında görünür ışığın yansımasının, Bragg kırınımı ile doğrudan ilişsisi kanıtlanmıştır [23-28]. Cam yüzeylere kaplanan ETPTA polimer yapının içerisine gömülü silika küreleri, hidroflorik asit ile seçici olarak uzaklaştırıldı.

Hidroflorik asit aşındırma sonrası; Şekil 6, üst tabaka kürelerin, sadece alt tabakada bulunan küreler tarafindan oluşturulan üçgen şeklinde düzenlenen boşlukların doldurulduğu oldukça düzenli bir yapıyı göstermektedir. Film kalınlığı boyunca altıgen olarak paketlenmiş katmanlar ve komşu katmanlar arasında paket yapılar, Şekil 5 ve Şekil 6 görüleceği üzere iyi derecede korunduğu açıktır. Daha yakın büyütmelere bakıldığında, orijinal nanokompozitler de bulunan silika kürelerin temas bölgelerinden kaynaklanan iç içe geçen gözenekler açıkça seçilebilmektedir (Şekil 6A ve 6B). Döner kaplamayla, kolloidal kristal eldesinin; hem kaymayla indüklenmiş dizilime; örneğin, kaplama hızı ve kaplama süresine(bkz. Şekil 7A ve 7B), hem de ardından monomer polimerizasyonuna bağlı olduğu gösterildi[29-33]. Şekil 8 de elde edilen çeşitli partikül çaplarına bağlı olarak farklı dalga boylarında kırmızı zirve noktaları normal beyaz 1şık altında ölçülmüş optik iletim spektrumlarıdır. Kırmızı eğri, kolloidal şablonların (ters makro gözenekli polimer filmler) gözenek boyutlarına bağlı olarak dalga boyu kayma değerini gösterir[33-35]. Bragg yasası ile iyi bir uyum içinde olan Şekil $8^{‘}$ de gösterilen liner kırmızı çizgi; boşluk çaplarına karşı $\lambda$ tepe noktası pozisyonlarına denk geldiği görüldü[17-20]. Bu çalışmanın sonuçları ve tartışma önerileri şu şekilde özetlenebilir. Bunlar; döner kaplama tekniğiyle bu zamana kadar gerçekleştirilen döner kaplama tekniklerinde sadece 3B kolloidal film ve şablonların üretimi başarılabilmiştir[1-8]. Bu teknik; kullanılarak klasik 3B kristal kaplama metotlarının [9-15] yerine, sadece önceki çalışmalarda kullanılmış süspansiyona \% 0.1 (v:v) etilen glikol eklenerek, cam yüzeyler üzerinde $6000 \mathrm{rpm}$ hızda, 2B (tek tabakalı) filmlerin elde edilebileceği kanıtlanmıştır(bkz. Şekil 5A ve 5B).

Elde edilen düzlemsel numuneler dönüş hızı ve süresinin değiştirilmesi ile kolayca ayarlanabilen oldukça düzgün bir kalınlığa sahip olduğu görülmüştür. Potansiyel elektronik devre ve optik kaplama uygulamaları için standart [15-16] yarı iletken mikrofabrikasyon teknikleri kullanılarak mikron ölçekli nanodizilimlerin oluşturulabileceği kanıtlanmıştır.

Monomer kırılma indisi n: 1.4689 ve silika kolloidleri kırılma indisi n: 1.42 olarak ele alındığında [17-19]; kolloidal stabiliteyi ve küreler arasındaki van der Waals etkileşimlerini azaltan etkenin, önceki çalışmalardan çıkan sonuçlara bakıldığında[19-23]; indis eşleşmesine bağlı olduğu söylenebilir.

$400 \mathrm{~nm}$ den büyük silika kürelerinin hızlı yerçekimi sedimantasyonu, yüksek kaliteli kristallerin yapımında ciddi sorunlara neden olmasından ötürü, önceki çalışmalarda uygulanmış yöntemler örneğin; elektrostatik, şablon kaplamaya[6,7] kıyasla, bu kaplama tekniğinin, bir başka avantaj sağladığıda görülecektir.

Jiang ve Bardosovanın meslektaşlarıyla daha önce ulaşmış oldukları sonuçlara dayanarak, ETPTA monomerinde silika partiküllerin sedimantasyon hızı, moleküler yapılar ve etkileşimler nedeniyle sudakinden yaklaşık 70 kat daha yavaş olduğu tespit edilmiştir[24-26].

Plazma aşındırma sürecinden sonra 30 mikron kalınlığındaki bir numune için silika kürelerin uzaklaştırılması, HF asit uygulamasıyla 24 saatden 10 dakikanın altına düştüğü görülmüştür. Bunu, üst kaplayıcı tabaka ETPTA polimerin, koruyucu bir tabaka olarak, HF asit çözeltisinin, kristal tabakalar arasına yayılımını engellediği söylenebilir.

Kimyasal özelliklerdeki önemli farklarından ötürü, ETPTA ve silisin her biri, diğerinin yapısını bozmadan, sırasıyla elde edilen kolloidal kristalden ve makro gözenekli polimer yapıdan seçici bir şekilde uzaklaştırılabilmiştir.

Oksijen plazma aşındırma; küreler ve çatlaklar gibi kusurlar ortaya çıkmaması nedeniyle silika partikülleri etkilenmemekte, bu nedenlede ETPTA polimer matrisinin çıkarılmasında kalsinasyondan daha iyi bir seçenek olduğu kabul edilebilir[27-29]. 
$\mathrm{Bu}$ çalışmada çıkan sonuçlardan biri ve Yang in çalışma grubunun elde etmiş olduğu sonuçlarıda destekleyen, düzlemsel kolloidal kristal şablonlar; hava, polimer fraksiyonunu değiştirdiğinden, kırılma indisi kontrast artışı nanokompozitlerin kendisinden daha güçlü Bragg kırınımı sergilediği gösterilmiştir[31].

SEM görüntüleri ve Şekil 3A daki Fourier dönüşümü, beklenen altıgen dizilimi desteklemektedir[30, 32, 33].

Kayma akışının atomik sistemlerin genel özellikleri üzerindeki etkisini araştırmak için değerli bir model olan kayma sıralı kolloidal kristalleşme; yoğun biçimde araştırıldı[33, 43]. Bu çalışmalardan çıkarılan sonuca göre; döner kaplama çalışmamızda, tipik makaslama oranları (> 105) olarak tespit edilmiştir.

2B altıgen olarak paketlenmiş kolloidal katmanların, santrifüj (F2r) ve viskoz $(-\eta(\partial 2 v / z 2 z))$ bağlanması nedeniyle kayar bir katman mekanizması ile $[34,36]$ açıklanabileceği görülmüştür.

Elde edilen tüm sonuçlar, bir bütün olarak değerlendirildiğinde, bu merkez kaç kuvvetine dayalı kristalleşme tekniğinin, oldukça düzgün kalınlık sağlaması, modern mikrofabrikasyon yöntemi ile uyumluluğu, düşük maliyet sunmasından ötürü, 2B nanodizilim teknoloji uygulamalarında rahatlıkla kullanılabileceği tespit edilmiştir.

\section{TEŞEKKÜR}

$\mathrm{Bu}$ proje, Harran Üniversitesi, BAP tarafindan, Biyomoleküllerin saflaştırılmasında kullanılacak manyetik nano partiküllerin üretimi başlıklı, 18206 nolu proje ile desteklenmiştir.

Ayrıca makalede destek veren Harran Üniversitesi Bölüm Mensuplarına teşekkür ederim.

\section{KAYNAKÇA}

[1] J. F. Bertone, P. Jiang, K. S. Hwang, D. M. Mittleman, and V. L. Colvin, "Thickness dependence of the optical properties of ordered silica-air and air-polymer photonic crystals," Physical Review Letters, vol. 83, no. 2, pp. 300-303, Jul 121999.

[2] J. H. Kim, S. H. Kang, K. Zhu, J. Y. Kim, N. R. Neale, and A. J. Frank, "Ni-NiO core-shell inverse opal electrodes for supercapacitors," Chemical Communications, vol. 47, no. 18, pp. 5214-5216, 2011.

[3] A. K. Samusev, K. B. Samusev, and M. V. Rybin, "Two-dimensional light diffraction from thin opal films," Physics of the Solid State, vol. 53, no. 5, pp. 1056-1061, 2011// 2011.

[4] H. Xing, J. Li, J. Guo, and J. Wei, "Bio-inspired thermal-responsive inverse opal films with dual structural colors based on liquid crystal elastomer," Journal of Materials Chemistry C, vol. 3, no. 17, pp. 4424-4430, 2015 2015.
[5] F. Malet, M. Pi, M. Barranco, E. Lipparini, and L. Serra, "Optical response of two-dimensional few-electron concentric double quantum rings: A local-spin-densityfunctional theory study," Physical Review B, vol. 74, no. 19, Nov 2006.

[6] S. Middleman, "The effect of induced air-flow on the spin coating of viscous liquids," vol. 62, ed: Journal of Applied Physics, 1987.

[7] Y. Ren and A. O. Adeyeye, "Magnetic spin states and vortex stability control in elongated $\mathrm{Ni}(80) \mathrm{Fe}(20)$ nanorings," Journal of Applied Physics, vol. 105, no. 6, Mar 2009.

[8] P. Jiang and M. J. McFarland, "Large-scale fabrication of wafer-size colloidal crystals, macroporous polymers and nanocomposites by spin-coating," Journal of the American Chemical Society, vol. 126, no. 42, pp. 1377813786, Oct 272004.

[9] C. A. F. Vaz et al., "Ferromagnetic nanorings," Journal of Physics-Condensed Matter, vol. 19, no. 25, Jun 2007.

[10] J. Wang, K. Deshpande, and G. B. McKenna, "Determination of the Shear Modulus of Spin-Coated Lipid Multibilayer Films by the Spontaneous Embedment of Submicrometer-Sized Particles," Langmuir, vol. 27, no. 11, pp. 6846-6854, Jun 72011.

[11] T. Yang, A. Hirohata, M. Hara, T. Kimura, and Y. Otani, "Current-induced vortex-vortex switching in a nanopillar comprising two Co nano-rings," Applied Physics Letters, vol. 90, no. 9, Feb 2007.

[12] K. Askar, B. M. Phillips, and Y. Fang, "Selfassembled self-cleaning broadband anti-reflection coatings," Colloids and Surfaces a-Physicochemical and Engineering Aspects, vol. 439, pp. 84-100, Dec 2013.

[13] T. Yang, A. Hirohata, L. Vila, T. Kimura, and Y. Otani, "Vertical stack of Co nanorings with currentperpendicular-to-plane giant magnetoresistance: Experiment and micromagnetic simulation," Physical Review B, vol. 76, no. 17 , Nov 2007.

[14] K. Askar and P. Jiang, "Self-assembled nanoparticle antireflection coatings on geometrically complex optical surfaces," Optics Letters, vol. 43, no. 21, pp. 5238-5241, 2018.

[15] A. Blanco and G. Hagy, "Large-scale synthesis of a silicon photonic crystal with a complete three-dimensional bandgap near 1.5 micrometres," Nature, 10.1038/35013024 vol. 405, no. 6785, pp. 437-440, 05/25/print 2000.

[16] T. Ding, K. Song, K. Clays, and C.-H. Tung, "Bottom-Up Photonic Crystal Approach with Top-Down Defect and Heterostructure Fine-Tuning," Langmuir, vol. 26, no. 6, pp. 4535-4539, Mar 162010.

[17] J. D. Joannopoulos, Photonic Crystals. 2000, 2011. [18] Y. Bao, H. Fong, and C. Jiang, "Manipulating the Collective Surface Plasmon Resonances of Aligned Gold Nanorods in Electrospun Composite Nanofibers," Journal of Physical Chemistry C, vol. 117, no. 41, pp. 21490-21497, Oct 172013.

[19] H. Yang and P. Jiang, "Large-Scale Colloidal SelfAssembly by Doctor Blade Coating," Langmuir, vol. 26, no. 16, pp. 13173-13182, 2013/12/25 2010. 
[20] H. Yang and P. Jiang, "Macroporous photonic crystal-based vapor detectors created by doctor blade coating," Applied Physics Letters, vol. 98, no. 1, pp. 011104011104-3, 2011.

[21] N. Gozubenli, E. Yasun, and L. Boskic, "Fabrication of nanoporous film by transfer of colloidal particles and application to biomacromolecules," Applied Nanoscience, vol. 8, no. 4, pp. 739-750, 2018/04/01 2018.

[22] H. Yang, N. Gozubenli, Y. Fang, and P. Jiang, "Generalized Fabrication of Monolayer Nonclose-Packed Colloidal Crystals with Tunable Lattice Spacing," Langmuir, vol. 29, no. 25, pp. 7674-7681, Jun 252013.

[23] X. Liu, B. Choi, N. Gozubenli, and P. Jiang, "Periodic arrays of metal nanorings and nanocrescents fabricated by a scalable colloidal templating approach," Journal of Colloid and Interface Science, vol. 409, pp. 52$58,11 / 1 / 2013$

[24] M. Bardosova, M. E. Pemble, I. M. Povey, and R. H. Tredgold, "The Langmuir-Blodgett Approach to Making Colloidal Photonic Crystals from Silica Spheres," Advanced Materials, vol. 22, no. 29, pp. 3104-3124, 2010.

[25] F. Burmeister, C. Schäfle, T. Matthes, M. Böhmisch, J. Boneberg, and P. Leiderer, "Colloid Monolayers as Versatile Lithographic Masks," Langmuir, vol. 13, no. 11, pp. 2983-2987, 1997/05/01 1997.

[26] P. Jiang, "Large-Scale Fabrication of Periodic Nanostructured Materials by Using Hexagonal Non-ClosePacked Colloidal Crystals as Templates," Langmuir, vol. 22, no. 9, pp. 3955-3958, 2013/12/25 2006.

[27] J. J. Kim, Y. Li, E. J. Lee, and S. O. Cho, "Fabrication of Size-Controllable Hexagonal Non-ClosePacked Colloidal Crystals and Binary Colloidal Crystals by Pyrolysis Combined with Plasma-Electron Coirradiation of Polystyrene Colloidal Monolayer," Langmuir, vol. 27, no. 6, pp. 2334-2339, Mar 152011.

[28] F. Caruso, H. Lichtenfeld, M. Giersig, and H. Mohwald, "Electrostatic self-assembly of silica nanoparticle - Polyelectrolyte multilayers on polystyrene latex particles," (in English), Journal of the American Chemical Society, Article vol. 120, no. 33, pp. 8523-8524, Aug 1998.

[29] A. M. Kalsin, M. Fialkowski, M. Paszewski, S. K. Smoukov, K. J. M. Bishop, and B. A. Grzybowski, "Electrostatic Self-Assembly of Binary Nanoparticle Crystals with a Diamond-Like Lattice," Science, vol. 312, no. 5772, pp. 420-424, 2006.

[30] Z. Ren, X. Li, J. Zhang, W. Li, X. Zhang, and B. Yang, "Tunable two-dimensional non-close-packed microwell arrays using colloidal crystals as templates," Langmuir, vol. 23, no. 15, pp. 8272-8276, Jul 172007.

[31] X. Yan, J. M. Yao, G. A. Lu, X. Chen, K. Zhang, and B. Yang, "Microcontact printing of colloidal crystals,"
Journal of the American Chemical Society, vol. 126, no. 34, pp. 10510-10511, Sep 12004.

[32] S. J. Ding, C. L. Zhang, M. Yang, X. Z. Qu, Y. F. $\mathrm{Lu}$, and Z. Z. Yang, "Template synthesis of composite hollow spheres using sulfonated polystyrene hollow spheres," Polymer, vol. 47, pp. 8360-8366, 2006.

[33] P. Jiang, J. F. Bertone, K. S. Hwang, and V. L. Colvin, "Single-crystal colloidal multilayers of controlled thickness," Chemistry of Materials, vol. 11, no. 8, pp. 21322140, Aug 1999.

[34] A. P. Bartlett, M. Pichumani, M. Giuliani, W. Gonzalez-Vinas, and A. Yethiraj, "Modified Spin-coating Technique to Achieve Directional Colloidal Crystallization," Langmuir, vol. 28, no. 6, pp. 3067-3070, Feb 142012.

[35] L. Li, T. Y. Zhai, H. B. Zeng, X. S. Fang, Y. Bando, and D. Golberg, "Polystyrene sphere-assisted onedimensional nanostructure arrays: synthesis and applications," (in English), Journal of Materials Chemistry, Article vol. 21, no. 1, pp. 40-56, 2011.

[36] P. Jiang and M. J. McFarland, "Wafer-Scale Periodic Nanohole Arrays Templated from TwoDimensional Nonclose-Packed Colloidal Crystals," Journal of the American Chemical Society, vol. 127, no. 11, pp. 37103711, 2013/12/25 2005.

[37] M. Giuliani, W. Gonzalez-Vinas, K. M. Poduska, and A. Yethiraj, "Dynamics of Crystal Structure Formation in Spin-Coated Colloidal Films," Journal of Physical Chemistry Letters, vol. 1, no. 9, pp. 1481-1486, May 62010. [38] A. Budkowski et al., "Polymer blends spin-cast into films with complementary elements for electronics and biotechnology," Journal of Applied Polymer Science, vol. 125, no. 6, pp. 4275-4284, Sep 152012.

[39] S. L. Burrs et al., "A comparative study of graphene-hydrogel hybrid bionanocomposites for biosensing," Analyst, 10.1039/C4AN01788A vol. 140, no. 5, pp. 1466-1476, 2015.

[40] H. Jiang, K. Yu, and Y. Wang, "Antireflective structures via spin casting of polymer latex," Optics Letters, vol. 32, no. 5, pp. 575-577, 2007/03/01 2007.

[41] X. Li et al., "Modulating Two-Dimensional NonClose-Packed Colloidal Crystal Arrays by Deformable Soft Lithography," Langmuir, vol. 26, no. 4, pp. 2930-2936, Feb 162010.

[42] P. Jiang, T. Prasad, M. J. McFarland, and V. L. Colvin, "Two-dimensional nonclose-packed colloidal crystals formed by spincoating," Applied Physics Letters, vol. 89, no. 1, Jul 32006.

[43] T. S. Juliane Junesch, and A. B. Dahlin, "Optical Properties of Nanohole Arrays in Metal-Dielectric Double Films Prepared by Mask-on-Metal Colloidal Lithography," vol. 6 (11),, ed: ACS Nano, 2012, pp. 10405-10415. 